\title{
Recessive dystrophic epidermolysis bullosa, generalized intermediate
}

INSERM

\section{Source}

INSERM. (1999). Orphanet: an online rare disease and orphan drug data base. Recessive dystrophic epidermolysis bullosa, generalized intermediate. ORPHA:89842

Recessive dystrophic epidermolysis bullosa (RDEB)-generalized other, also known as RDEB non-Hallopeau-Siemens type, is a subtype of DEB (see this term) characterized by generalized cutaneous and mucosal blistering that is not associated with severe deformities. 